\title{
Study on the Application of Lu Brocade patterns on Modern Clothing
}

\section{Design}

\author{
Ying Dai ${ }^{1}$ \\ ${ }^{1}$ Jiangxi Institute of Fashion Technology, Nanchang, Jiangxi, 330201
}

KEYWORDS: Lu Brocade; Costume Design; Patterns; Folk Textiles; Molding Patterns

\begin{abstract}
In our country there are a lot of folk textile art works with a strong folk characteristic, $\mathrm{Lu}$ Brocade is one of the best and one of its biggest feature is the pattern. This paper on for several He Gezi pattern brocade constitute characteristics for analysis and research, while maintaining its essence the same pattern, some brocade patterns applied to modern fashion design. After a series of practice can be proved, though brocade patterns will be applied directly to the design relatively easy to operate, it also has national characteristics, but copy and paste traces too obvious. So now brocade patterns use more indirect way into modern design. This approach also makes it easier for people to accept.
\end{abstract}

\section{Introduction}

Brocade produced in Heze and Jining area called Lu Brocade. It is known as eight-four-frame tie or tie. Usually women, Textile yarn dyed color, then use the color of soil loom woven cotton fabric. [1]. Lu Brocade exists long ago, has a very long history, it is one of Shandong Province, the traditional material heritage. [2]. Shandong Province in 1984 to carry out work on protection against brocade [3]. According to the "Juan County" records, Lu Brocade Shandong Province Development Research Group in September 1984 was formally established and the Panel extensive mining folk patterns and related brocade for new product development, before and after sorting out the more than eight hundred kinds of pattern styles develop new products about 90 kinds. These patterns are widely used in tourism, clothing and home decorative items [4] "This paper will put Yang Lu Brocade for application in modern fashion design to discuss.

\section{The Cultural Lu Brocade}

Belonging to a folk brocade textile articles, which has a very deep historical deposition associated with significant national identity, the Yellow River is the essence of cultural development, is the brainchild of Shandong residents' daily life and the general portrayal of women compatriots. First, the unique geographical environment of Shandong Lu Brocade provides a rich resource. Secondly, the local advanced textile technology development has brought to Lu Brocade technical support. Again, brocade with local residents toil textile has a very close relationship, from the Tang Dynasty, Du Fu, "Yi Xi" "Qi Wan Lu stripe car classes, farming men and women weave not phase loss," Li Bai and "May the Dong Lu, , A. Wenshang Jun "and" Lu people do heavy weaving, loom Ming curtain cage, "the poem can appreciate. After the Qing Dynasty, textile equipment and technology to do the continuous improvement and rich brocade textile techniques rose to a very sophisticated level, at the time it is society as a tribute into the royal special items. 
And other similar folk art, Lu Brocade is artistic culture is used as a transfer medium, showing the traditional cultural environment has aesthetic concepts and modeling. In brocade weaving process, local women have formed a unique ideology. Lu Brocade on the general use of contrast color display, which allows people to find wealth in the rustic, simple seen in gorgeous images. Their usual colors are black, white, red, pink, rosy red, green, green, indigo, blue, yellow, light yellow, coffee. According to traditional moral culture, Lu Brocade has certain formal requirements in color with the expression, such as with the "black + red + purple + green + yellow + blue + white" performance "co bucket pattern" pattern, with "Mei + red + purple + orange + black light yellow "performance" colorful river "and other patterns. Among them, black and white are two very important color, and use them to experience those with very bright colors, which is thousands of years in Shandong residents toiling constantly accumulated.

Folklore is a resident of the development, production, transfer and want the presence of social production and the life of a culture used. Difference because it has the heritage of the space, as well as regional and national exhibits relatively significant personality differences. In Lu Brocade wen spread of limited space, Shandong women not only to achieve the recognition and enhance the value of its own location in the family life, but also show their thoughts and feelings. Lu Brocade graphics and color, to show the life habits of residents in Shandong Qilu, emotional, aesthetic ideas and work rules, is being spread a culture medium Qilu materialized.

\section{Second, The Characteristics Of Traditional Brocade Patterns}

On the structure, the brocade pattern forming elements is a geometric shape, Table 1 gives it its shape analysis. Early most general geometry is twill, stripes there Plaid these three. They are relatively simple in the production and distribution, so the use of time is relatively long. Wherein the strip has been applied to the grid with brocade development process, it may also be called common graphical brocade. And the time to produce special patterns of late, textile technology is more complex, rich type. What is special pattern, that is, which possesses allegorical folk patterns. They can be reflected in the name of it, such as dates lines, waterlines, dog tooth pattern, bucket grain, sesame pattern, combined bucket Wen, Wen eyes geese, cats and other hoofed patterns. It is easy to see that they are all derived from plants and animals to people's daily life and labor of the problems encountered, etc. [5]. On the structure, the special patterns of evolution and development of conventional fact patterns, that is, under the premise of regular patterns, the use of the formation of some form of superposition rich graphics.

\section{A direct application in fashion design}

Lu Brocade loft in the current fashion design is not the most simple use of brocade patterns make no changes to its full use in the clothing or styling. Overall Operation Mode refers to the use brocade patterns to be processed into a complete garment. On the above described content, brocade pattern is generally a two-way or four uninterrupted geometry, have some uninterrupted and relatively symmetrical these two characteristics, so that the patterns of use to large brocade garments now in the visual the first to scale patterns, and continuous symmetry, as well as clothing and modern multi-cut link between the sheet and the three-dimensional shaping planning, Wherein the contact can show up in the demo. The combined use of the bucket to the front lines in the form of double-slit skirt type A (see Figure 1). It can be seen that the slits configured to break the patterns have integrity, humanity at the beginning if not quickly viewing patterns to do a whole judge, then the clothing will let you produce a very confusing visual effects. Figure 2 shows the patterns of use 
in the years more than the dress. Although it has the form of a fixed breakthrough in one sense and with effective use between pieces and patterns, it has the patterns into a center-point of symmetry of the V-shaped lines. Not only has the traditional patterns that have structural features, but also to meet the current demand for clothing three-dimensional modeling, to produce a more complete use of scientific structure.

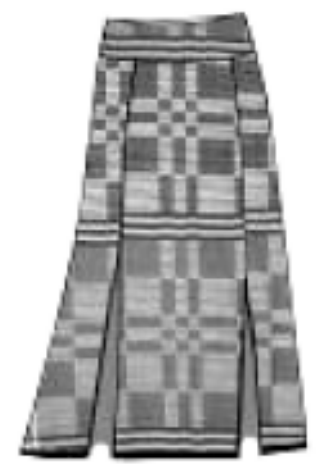

Fig. 1 Application of combined grain bucket

The brocade pattern is the topical application of local design in the way of clothing. In this design, only with brocade patterns on the local details of clothing, the other part is used to make other fabric stitching design. In general, the use of the local decorative collar, cuffs, cuffs and hem, etc. [6].

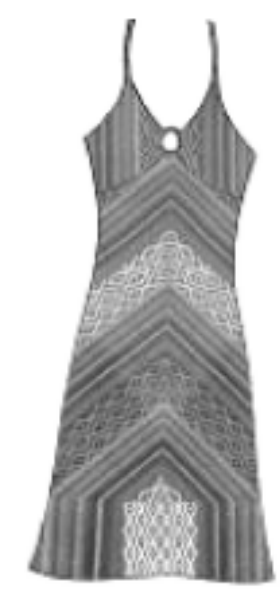

Fig. 2 Perennial grain surplus application

In a piece of clothing, the most concern is the neckline, so the designer, design neckline success is directly related to the success or failure of this dress [7]. So in the design of the collar will need to carefully consider two aspects, one is to be designed according to the structure of the collar, there is usually a basic collar type, namely, no collar, stand-up collar and lapel. All other areas are formed in the evolution of these three basic shape of the collar comes. Moreover, because less material at the collar of the whole dress and if the collar brocade, it also stands out with strong ethnic characteristic. Figure 3 below shows the close fight is the text used in the collar. 


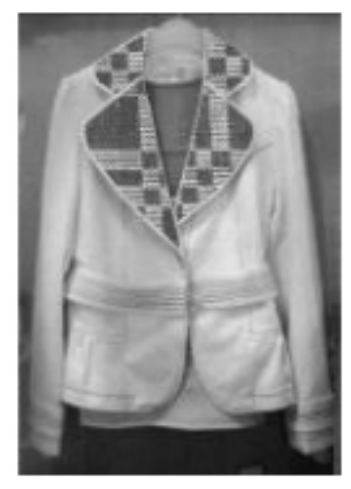

Fig. 3 Close fight application pattern in the collar

In the whole dress, the pocket is the most flexible material. In general, there are pockets of decorative, practical and decorative practical combination of three. And as the pocket is concerned, it is not limited whole garment structure, so it can have many variations. Usually there are pockets of pocket, patch pocket, cover bags and bags of four. It is because of the diversity and randomness pocket, so it can be applied to the brocade pocket, but in operation, and must pay attention to their zippers, buttons, etc. with the use. 4 is a close fight on land in the pocket of the application below.

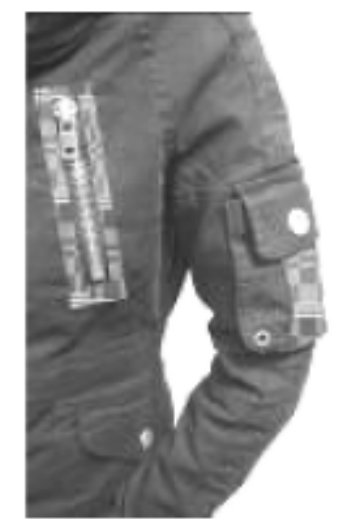

Fig.4 Close fight application pattern in pocket

An excellent clothe must have distinctive details and decorative features laundry belt is also common. Belt used properly, can bring a good image. In the brocade patterns, and mostly continuous elongated square pattern, and such patterns, especially suitable for the belt, so it is possible to use the brocade belt so extraordinarily well on the belt. 5 is the date the application pattern in the figure below the belt.

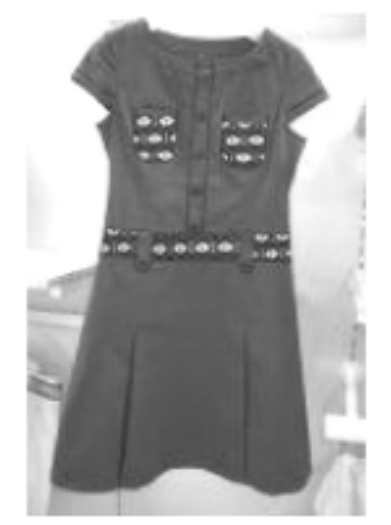

Fig. 5 Date pattern used in the belt 


\section{Conclusion}

To sum up, brocade patterns applied directly to clothing is the most easy to grasp way. However, the application of brocade pattern in the clothing as a whole, must pay more attention to their symmetry features, although some patterns can be changed in the application of its symmetry, but it must not undermine the continuity of the pattern itself. If it is applied brocade patterns on the details of clothing, you do not need too much to consider this one, only the need to strengthen and coordinate their echoes and other applications on the fabric.

\section{Reference}

[1] Qian Xin, Zhao Meng. Brocade Patterns [J]. In the Modern Fashion Design Textile Sinica, 2010,01: 96-101 + 107 .

[2] Zhang Yi. Research Folk Art In The Modern Interior Fabric In [D]. Shandong Jianzhu University, 2013.

[3] Yang Yongqing, Yan Qi, Lu Brocade. Cultural and Application of Innovation and Research, [J] Silk, 2011,11: 45-49.

[4] Li Rui. The Inheritance and Development of Brocade Weaving Skills of Research [D]. Shandong University, 2010.

[5] Sun Chengcheng. Dynasty Court Dress Patterns Applied Research in Haute Couture in The [D]. Beijing Institute of Clothing, 2012.

[6] Zhang Lingling. Research Lu Brocade Art in The Interior Soft Furnishings Design [D].Central South University of Forestry And Technology, 2011. 